\title{
A Strain Energy Filter for 3D Vessel Enhancement
}

\author{
Changyan Xiao ${ }^{1,2}$, Marius Staring ${ }^{1}$, Denis Shamonin ${ }^{1}$, Johan H.C. Reiber ${ }^{1}$, \\ Jan Stolk ${ }^{1}$, and Berend C. Stoel ${ }^{1}$ \\ ${ }^{1}$ Division of Image Processing, Department of Radiology, Leiden University Medical \\ Center, P.O. Box 9600, 2300 RC, Leiden, The Netherlands \\ ${ }^{2}$ College of Electrical and Information Engineering, Hunan University, China
}

\begin{abstract}
The traditional Hessian-related vessel filters often suffer from the problem of handling non-cylindrical objects. To remedy the shortcoming, we present a shape-tuned strain energy density function to measure vessel likelihood in 3D images. Based on the tensor invariants and stress-strain principle in mechanics, a new shape discriminating and vessel strength measure function is formulated. The synthetical and clinical data experiments verify the performance of our method in enhancing complex vascular structures including branches, bifurcations, and feature details.
\end{abstract}

\section{Introduction}

Pulmonary vessel detection plays an important role in computer analysis of lung CT images. Evaluating vessels is of considerable value to diagnosing for example pulmonary emboli and hypertension. Additionally, there is an interest in identifying the vascular trees as landmarks for matching lungs across variety 1. However, accurate and robust detection of pulmonary vessels still remains a problem because of the geometrical complexity and fine characteristics of details. Especially, with non-contrast-enhanced images being widely used in CT densitometry, the vascular detection becomes even more challenging.

In this paper, we present a 3D vessel enhancing filter with the main purpose to break the cylinder limits of traditional Hessian filters 2345], improve their ability in preserving more general vascular structures like bifurcations. The method is initially motivated by a recent achievement of stress and strain theory in solid mechanics [6], which was introduced previously to fMRI imaging by Ennis et al. 7. We establish the idea basically on a link between image structures and locally compressed states of material, due to the mathematical equivalence between the image Hessian matrix and the mechanical stress or strain tensor.

\section{Theoretical Background}

The Hessian matrix $H$ is equivalent to the stress tensor in solid mechanics in that it is also a second-order symmetrical tensor. Then, $H$ can be decomposed into

T. Jiang et al. (Eds.): MICCAI 2010, Part III, LNCS 6363, pp. 367-374, 2010.

(C) Springer-Verlag Berlin Heidelberg 2010 
an eigensystem of three real eigenvalues $\lambda_{i}$ and three orthogonal eigenvectors $\boldsymbol{e}_{i}$ $(i=1,2,3)$.

A common decomposition of stress tensor is $H=\bar{H}+\widetilde{H}$, where $\bar{H}$ corresponds to the so-called "isotropic" or spherical component. $\widetilde{H}$ indicates the "deviatoric" or anisotropic component, and contains the directional inequality information. They are calculated as

$$
\bar{H}=\frac{1}{3} \operatorname{tr}(H) \mathbf{1}=\frac{1}{3}(H: \mathbf{1}) \mathbf{1}, \quad \widetilde{H}=H-\frac{1}{3} \operatorname{tr}(H) \mathbf{1}=H-\frac{1}{3}(H: \mathbf{1}) \mathbf{1},
$$

where $\mathbf{1}$ is the identity matrix and the colon $(:)$ denotes the tensor contraction operator. According to the stress principle, only the isotropic component causes volume changes, while the deviatoric one exclusively accounts for the distortion or shear without volume changes. Since $\bar{H}: \widetilde{H}=0$, the two components are mutually orthogonal. From this, Criscione et al. [6] introduced a set of tensor invariants $K_{i}$ :

$$
K_{1}=\operatorname{tr}(H), K_{2}=\|\widetilde{H}\|_{F}, \text { and } K_{3}=3 \sqrt{6} \operatorname{det}\left(\widetilde{H} /\|\widetilde{H}\|_{F}\right),
$$

where $\operatorname{det}()$ is the determinant operator. Due to orthogonal decomposition, the three invariants reflect mutually independent properties of local deformation. The intuitive physical meaning behind them is that $K_{1}$ represents the amount of dilatation (with a negative value corresponding to compression), $K_{2}$ the magnitude of distortion, and $K_{3}$ the mode or type of distortion.

\section{Method}

The development of our vessel filter mainly originates from the above stress tensor invariants, and is divided into five parts:

\subsection{Measure of Brightness Contrast}

We first develop a contrast term from the invariance $K_{1}$. Since $K_{1}$ is a measure of local volume change, it indirectly reflects the density variation inside an infinitesimal element of material by the mass conservation law. If the density is assumed to be the image intensity, $K_{1}$ will be an indicator of relative intensity change, i.e. brightness contrast.

Generally, we only need to confine the sign of $K_{1}$, with negative (positive) values corresponding to bright (dark) objects. For pulmonary CT images, we add a relative threshold in proportion to the maximum magnitude of eigenvalues $\left(\lambda_{m}\right)$ to ensure noise immunity, i.e.

$$
\left|K_{1}\right|=\left|\lambda_{1}+\lambda_{2}+\lambda_{3}\right|>3 \alpha \lambda_{m}
$$

Here, the parameter $\alpha>0$ is used to adjust sensitivity. 


\subsection{Measure of Structure Strength}

In mechanics, the strain energy density is a term to quantify the local energy stored in solids due to stress effects after mechanical loading. Since the procedure simultaneously results in an uneven mass distribution, the strain energy also indirectly reflects the material density inhomogeneity. Based on the similarity of density variation to intensity concentration around image structures, we introduce an strain energy density term to measure structure strength.

Consider an isotropic and linear elastic material, the strain energy density is defined as $U=\frac{1}{2} \sum_{i=1}^{3} \sum_{j=1}^{3} \sigma_{i j} \eta_{i j}$, with $\sigma_{i j}$ and $\eta_{i j}$ the elements of stress and strain tensors. We rewrite it in the form of orthogonal components of the stress tensor: $U(H)=\frac{1-2 \nu}{2 \epsilon}(\bar{H}: \bar{H})+\frac{1+\nu}{2 \epsilon}(\widetilde{H}: \widetilde{H})$. Here, $\epsilon$ and $\nu$ are Young's modulus and Poisson's ratio, respectively. Thus, the energy density function is divided into two independent parts: volume changing (isotropic) energy and distortion deforming (anisotropic) energy. We further omit the fixed $\epsilon$, and adopt the square root to keep the same power order of the original intensity, i.e.

$$
\rho(H)=\sqrt{(1-2 \nu)(\bar{H}: \bar{H})+(1+\nu)(\widetilde{H}: \widetilde{H})} .
$$

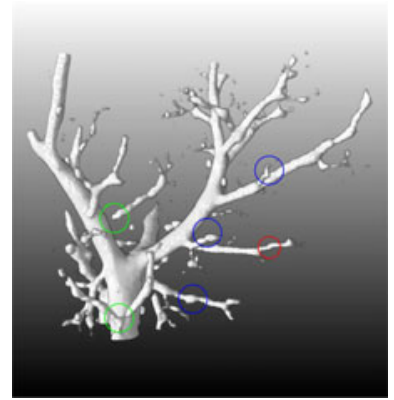

(a) $\nu=-1.0$

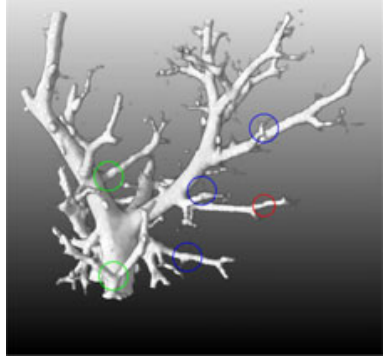

(b) $\nu=0.0$

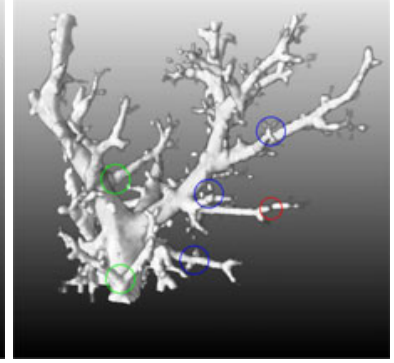

(c) $\nu=0.5$

Fig. 1. Vascular structure strength measure, see eq.(7), with varying parameter $\nu$

Notice the Poisson's ratio $\nu \in[-1,0.5]$ essentially describes the mutual influence between deformations in different directions. For image processing, if $\nu<0$, the intensity concentrating in one direction will cause the relative intensity to increase in the perpendicular directions, which is known to generate isotropic "blob" structures. Inversely, $\nu>0$ will adversely affect the intensity concentration in orthogonal directions, and then encourages anisotropy. If $\nu=0$, we have $\rho(H)=\sqrt{\lambda_{1}^{2}+\lambda_{2}^{2}+\lambda_{3}^{2}}$, which corresponds to the "second order structureness" used by Frangi et al. 4]. We verified the effect of $\nu$ through a real image experiment in Fig.1. It can be found that a low $\nu$ tends to develop smooth surfaces, the anisotropic structures (labeled with "green" circles) and feature details ("blue" circle) are gradually enhanced with increasing $\nu$. However, a very large $\nu$ is at the risk of exaggerating blob-like deformations ("red" circle). 


\subsection{Intensity Continuity Constraint}

To understand the Hessian eigenvalue distribution of general vascular shape, we introduce an intensity continuity constraint. As observed, most vessel structures including the branch and junction, share one common characteristic of intensity continuity, i.e. there exists at least one direction in which the intensity variation is very small. The intensity continuity at pixel $x_{0}$ in direction $\boldsymbol{r}$ is equivalent to $I_{\sigma}\left(x_{0}+h \boldsymbol{r}\right)-I_{\sigma}\left(x_{0}\right) \approx 0$, with $\sigma$ the observing scale, $\boldsymbol{r}$ a unit vector and $h$ the magnitude. Then, the corresponding Taylor expansion is $\nabla I_{\sigma}\left(x_{0}\right) \cdot \boldsymbol{r}+$ $\frac{h}{2} \boldsymbol{r}^{t} H_{\sigma}\left(x_{0}\right) \boldsymbol{r} \approx 0$. If the first-order derivative is negligible, the Hessian term will mainly account for the intensity variation, i.e. $\boldsymbol{r}^{t} H_{\sigma}\left(x_{0}\right) \boldsymbol{r} \approx 0$.

To ensure the dominance of the Hessian term, we additionally define a relative Hessian strength function

$$
G_{r e l}(x)=\exp \left(-\beta \frac{\|\nabla I\|}{\lambda_{m}}\right) .
$$

Here, $\beta>0$ is used to adjust the sensitivity of response. $G_{r e l}(x)$ will tend to 1 (or 0), while the gradient (or Hessian) term is negligible. This relative strength function is useful in suppressing step edges, which also responds strongly to eigenvalue detection like the second-order structures, but takes a strong gradient.

Based on the continuity constraint, the Hessian eigenvalue relation can be formulated as $c_{1}^{2} \lambda_{1}+c_{2}^{2} \lambda_{2}+c_{3}^{2} \lambda_{3}=0$, where $c_{i}$ indicate the coefficients of $\boldsymbol{r}$ under the eigen-system. The potential meaning is not only to explain the eigenvalue distribution of traditional line and sheet shapes, but also understand more general vascular structures like bifurcations and stenoses. The latter are then verified to have non-zero and differently signed eigenvalues.

\subsection{Vessel Shape Discrimination}

A vascular shape discriminating function can be formulated by combining the above tensor invariants. The mode of distortion (invariant $K_{3}$ ) provides a natural measure of shape anisotropy. But its discriminating ability will gradually decrease when the anisotropic extent becomes weaker. To remedy this drawback, we introduce the fractional anisotropy $F A=\sqrt{3(\widetilde{H}: \widetilde{H}) /(H: H)}$, which is actually a ratio of invariant $K_{1}$ and $K_{2}$, as an additional indicator. Here, a constant 3 is added to ensure that $F A=1$ for an ideal vessel branch $\left(\left|\lambda_{1}\right| \approx 0\right.$ and $\lambda_{2} \approx \lambda_{3} \gg 0$ or $\ll 0$ ).

Generally, FA and mode both measure the shape variety, but have different sensitivity as a function of anisotropy. Based on their response curves, we merge them in the final vessel shape discriminating function:

$$
V(x)=\left\{\begin{array}{ll}
\sqrt{F A}, & F A<1 \\
\frac{1}{2}[\operatorname{mode}(x)+1], & \text { otherwise }
\end{array} .\right.
$$

It can be verified that $V(x)$ approaches 0 for blob $\left(\lambda_{1} \approx \lambda_{2} \approx \lambda_{3} \gg 0\right.$ or $\left.\ll 0\right)$ and sheet shapes $\left(\lambda_{1} \approx \lambda_{2} \approx 0\right.$ and $\lambda_{3} \gg 0$ or $\left.\ll 0\right)$, whereas it takes 1 for line shapes 
corresponding to vascular branches. The most attractive property is that $V(x)$ also responds with high values to locally deformed structures like junctions and stenoses, which for example may have one positive and two negative eigenvalues.

\subsection{Multi-scale Vesselness Measure}

To generate a shape-tuned strain energy function, we combine the previous vascular structure related terms as

$$
\varphi(\sigma, x)=\left\{\begin{array}{l}
0, \text { if } \frac{1}{3}\left(\lambda_{1}+\lambda_{2}+\lambda_{3}\right)>-\alpha \lambda_{m} \\
\exp \left(-\beta \frac{\|\nabla I\|}{\lambda_{m}}\right) V^{\kappa}(x) \rho(H, \nu), \text { else. }
\end{array}\right.
$$

The power coefficient $\kappa \in[0,1]$ of $V(x)$ is added to adjust the sharpness of shape selectivity. The details of multi-scale integration can be referred to [34]. We also adopt the $\gamma$-normalization and rescaling operation to compensate intensity changing across scales.

\section{Experimental Results}

The proposed method is validated by using synthetical and clinical datasets. The filtering results were compared with three traditional Hessian-based filters 34,8 . The quantitative evaluation is conducted by calculating the precision - recall (PR) curves after binarizing the filtered images with different global thresholds. Two versions of recall (sensitivity) are defined, namely in terms of volume and skeleton of true vessels. The latter is specifically designed to emphasize the presence of vessels and neglecting its radial size, which is uncertain for a vessel with a Gaussian profile. The free parameters of all methods are optimized, based on the area under curve (AUC) of the PR curves.

\subsection{Synthetic Data}

As shown in Fig. 2(a), a digital phantom was generated with six objects to simulate different kinds of vessel structures, which resemble stenoses, varying diameters, bifurcations, and curved or touching branches.

We compared the four methods on the synthetic image with $16 \%$ variance noise in Fig. 2(b). The scale range used in this experiment is $1-6$ pixels, and is further divided logarithmically into 10 steps. The parameters of our proposed method were optimized to $\alpha=0.2, \beta=0.02, \kappa=0.4, \nu$ was set to 0 without priority to specific structures. The filtering results are given in Fig. 2(c)-(f). As expected, the traditional Hessian filters were not good in preserving the junctions and local deformations, where disconnections in varying degrees can be observed. The proposed method enjoyed more merits in enhancing both vascular branches and bifurcations. Additionally, detailed features like thin vessels and local diameter variety are well preserved. For quantitative evaluation, we refer to Fig. 3. The performance of our method belongs to the best two with Frangi's 


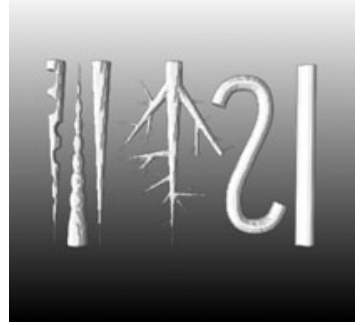

(a)

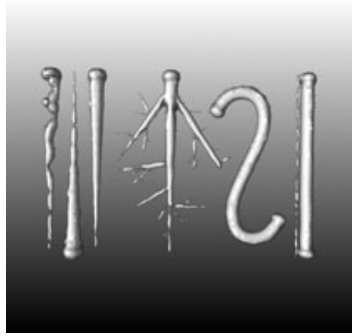

(d)

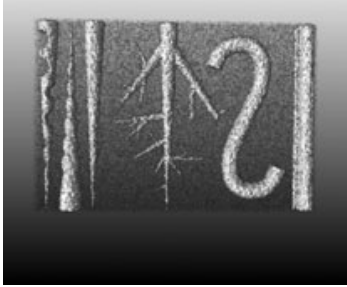

(b)

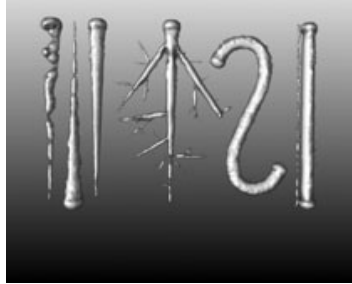

(e)

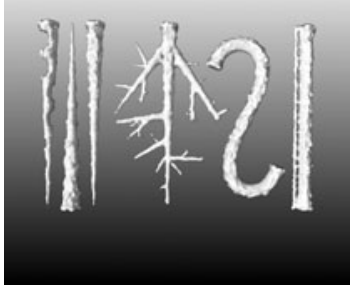

(c)

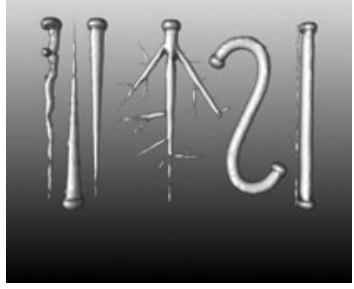

(f)

Fig. 2. Synthetic dataset experiment. (a) Original image, (b) synthetic image, enhanced images from (c) the proposed method, (d) Frangi [4],(e) Li [9], and (f) Sato [3] filters

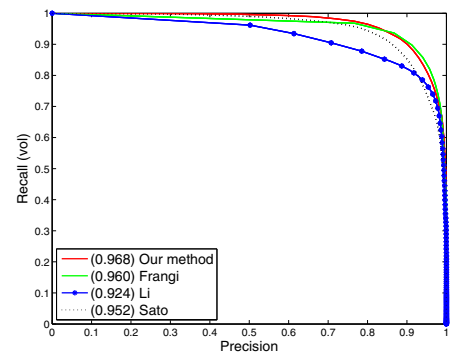

(a)

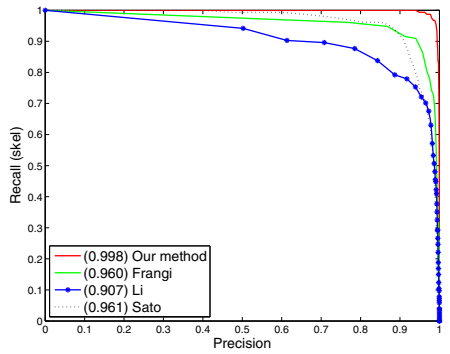

(b)

Fig. 3. PR curves of filtered synthetic images. (a) and (b) respectively correspond to the volume- and skeleton-based recall, where the numbers in legends are AUCs.

filter on the volume-based PR measure, while our advantage is dominant on the skeleton-based one. It can be understood from the distribution and AUCs of PR curves that our result takes higher completeness (recall) under the majority of volume fidelity (precision) levels compared with the other three methods. This just reflected the merit of our method in preserving thin vessels and connectivity of vascular networks. 


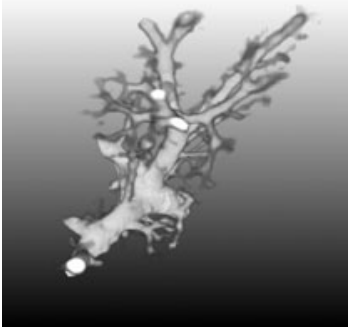

(a)

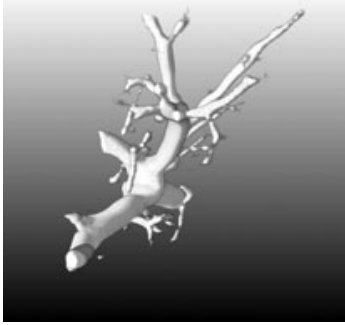

(d)

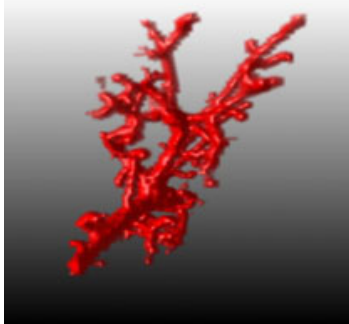

(b)

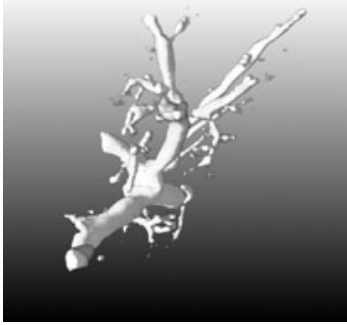

(e)

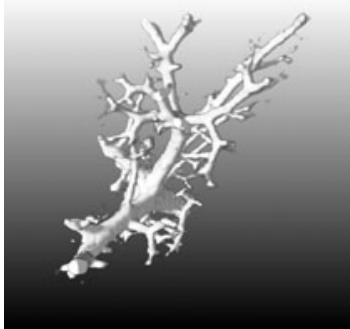

(c)

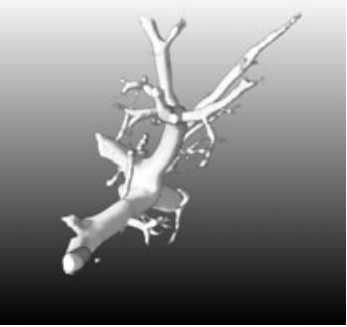

(f)

Fig. 4. Non-contrast enhanced pulmonary CT dataset experiment. (a) original image; (b) manually segmented "ground truth"; (c)-(f) give in order the filtered images from the proposed, Frangi 4 , Li 8 ] and Sato 3 methods

\subsection{Clinical Data}

Our method was further validated by using a cropped clinical pulmonary CT dataset. The images were acquired without contrast media injection, and the resolution was $0.7 \times 0.7 \times 0.5 \mathrm{~mm}$. The parameters of our filter were optimized to $\alpha=0.1, \beta=0.06$ and $\kappa=0.5$, and a positive $\nu=0.10$ was used to enhance junctions. The selected vascular scales were $0.5-3.0 \mathrm{~mm}$, and 10 steps were used.

For better observation of details, a region of interest was extracted and shown in Fig. 4(a), together with a manually segmented "ground truth" by experts in Fig. 4 (b) for reference. The extraction was drawn first by a radiologist, then a pulmonologist and a surgeon were asked to verify it. Fig. 4(c)-(f) are the corresponding results of the four filters. It is clear that the traditional filters improve the visualization of main vascular branches at the cost of weakening junctions and details. Our method keeps most vascular structures while suppressing the unwanted noise. Both branches and bifurcations are enhanced without distortion. In particular, thin vessels and details are clearly preserved with the continuity to main vessels. The merits can be further verified by the quantitative evaluation in Fig. 5 . 


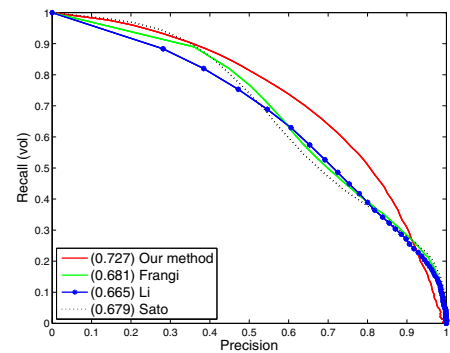

(a)

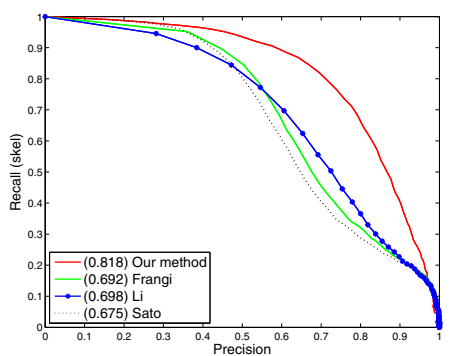

(b)

Fig. 5. PR curves of filtered clinical images. (a) and (b) respectively correspond to the volume- and skeleton-based recall with AUCs shown in the legends

\section{Conclusions}

In this paper, we have presented a $3 \mathrm{D}$ vessel enhancing filter based on the tensor invariants and strain energy density theory. The main feature is that we directly generalized the Hessian-based vesselness filters to non-tubular shapes and realized the enhancement of anisotropic vascular structures like junctions. The preliminary results verified the performance of our model in preserving locally deformed vessels and detailed features.

Acknowledgements. This work was funded by STW (grant LPG.07998) of the Netherlands and NSFC (No. 60835004, 60871096, 60872130) of China.

\section{References}

1. Sluimer, I., et al.: Computer analysis of computed tomography scans of the lung. TMI 25(4), 385-405 (2006)

2. Lorenz, C., et al.: Multi-scale line segmentation with automatic estimation of width, contrast and tangential direction in $2 \mathrm{D}$ and $3 \mathrm{D}$ medical images. In: CVRMed/MRCAS 1997, pp. 233-242 (1997)

3. Sato, Y., et al.: 3d multi-scale line filter for segmentation and visualization of curvilinear structures in medical images. In: CVRMed/MRCAS 1997, pp. 213-222 (1997)

4. Frangi, A.F., et al.: Multiscale vessel enhancement filtering. In: Wells, W.M., Colchester, A.C.F., Delp, S.L. (eds.) MICCAI 1998. LNCS, vol. 1496, pp. 130-137. Springer, Heidelberg (1998)

5. Krissian, K., et al.: Model-based detection of tubular structures in $3 \mathrm{~d}$ images. CVIU 80(2), 130-171 (2000)

6. Criscione, J.C., et al.: An invariant basis for natural strain which yields orthogonal stress response terms in isotropic hyperelasticity. J. Mech. Phys. Solids 48(12), 24452465 (2000)

7. Ennis, D.B., Kindlmann, G.: Orthogonal tensor invariants and the analysis of diffusion tensor magnetic resonance images. Magn. Reson. Med. 55(1), 136-146 (2006)

8. Li, Q., et al.: Selective enhancement filters for nodules, vessels, and airway walls in two- and three-dimensional ct scans. Med. Phys. 30(8), 2040-2051 (2003) 